\title{
Proinsulin and acute insulin response independently predict Type 2 diabetes mellitus in men - report from 27 years of follow-up study
}

\author{
B. Zethelius ${ }^{1}$, L. Byberg ${ }^{1}$, C. N. Hales ${ }^{3}$, H. Lithell ${ }^{1}$, C. Berne ${ }^{2}$ \\ ${ }^{1}$ Department of Public Health and Caring Sciences / Section of Geriatrics, Uppsala University, Uppsala, Sweden \\ 2 Department of Medical Sciences, Uppsala University, Uppsala, Sweden \\ 3 Department of Clinical Biochemistry, Addenbrooke's Hospital, Cambridge, UK
}

\section{Abstract}

Aims/hypothesis. Defects in insulin secretion and insulin action, resulting in compensatory hyperinsulinaemia, are the major abnormalities in the development of Type 2 diabetes mellitus (Type 2 diabetes). The most frequently used conventional immunoreactive assays for insulin cross-react with proinsulin. In shortterm studies ( $<5$ years), proinsulin predicts the development of Type 2 diabetes. We studied, with a 27 -year follow-up, the longitudinal relationships between intact proinsulin, 32-33 split proinsulin, specific and immunoreactive insulin (IRI), acute insulin response (AIR) after an intravenous glucose load and the development of Type 2 diabetes in a population-based cohort of 50-year-old men.

Methods. Fasting peptide concentrations were measured in plasma samples, stored since 1970-73 using specific two-site immunometric assays. IRI was measured at baseline using radioimmunoassay. Associations between development of Type 2 diabetes and predictor variables, were analysed with logistic regression. Results are shown as odds ratios (ORs) with their $95 \%$ confidence intervals (CIs) for a one standard deviation increase in a predictor variable.

Results. Cumulative incidence of Type 2 diabetes was $33 \%$ over 27 years of follow-up. Intact proinsulin (OR, 1.57, CI, 1.16-2.14), and 32-33 split proinsulin (OR, 1.70, CI, 1.20-2.39) were associated with development of Type 2 diabetes, independent of AIR, adjusted for BMI and fasting glucose, whereas specific insulin was not (OR, 1.31, CI, 0.98-1.77), nor was IRI (OR, 1.25, CI, 0.96-1.63). Proinsulin and AIR interacted in the development of Type 2 diabetes $(p<0.05)$.

Conclusion/interpretation. Proinsulin predicts the development of Type 2 diabetes mellitus over a 27-year period. [Diabetologia (2003) 46:20-26]

Keywords Proinsulin, insulin, immunoreactive, body mass index, epidemiology, risk factors, prospective study.
Defects in insulin secretion and insulin action, resulting in compensatory hyperinsulinaemia, are the major abnormalities in the development of Type 2 diabetes mellitus $[1,2]$. It has been shown that insulin secreto-

Received: 18 June 2002 / Revised: 20 August 2002

Published online: 8 January 2003

C) Springer-Verlag 2003

Corresponding author: B. Zethelius MD, Department of Public Health and Caring Sciences / Section of Geriatrics, Uppsala University, Box 609, 75125 Uppsala, Sweden, E-mail: bjorn. zethelius@pubcare.uu.se

Abbreviations: AIR acute insulin response; IRI immunoreactive insulin. ry dysfunction and insulin resistance, analysed by different clamp techniques, were independent predictors of worsening of glucose tolerance [3].

In several prospective studies, impaired insulin secretion assessed by a low acute insulin response (AIR) to a glucose load, was shown to be a predictor of the development of Type 2 diabetes [2, 4, 5, 6]. Furthermore, fasting hyperinsulinaemia indicative of insulin resistance is a predictor of Type 2 diabetes $[6,7,8]$. However, this association is less strong in the impaired than in the normal glucose tolerance state $[9,10]$. A complication in earlier studies is that conventional radioimmunoassays for insulin, measuring immunoreactive insulin (IRI), cross-react with proinsulin. 
Proinsulin has been shown to predict the onset of Type 2 diabetes in subjects with normal or impaired glucose tolerance in studies with follow-up time less than 7 years $[11,12,13,14,15,16]$. Both proinsulin and specific insulin are inversely associated with insulin sensitivity and positively associated with the AIR in subjects with normal glucose tolerance [17] and in subjects with Type 2 diabetes [18]. The roles of proinsulin, insulin resistance and impaired insulin secretion in the development of Type 2 diabetes over longer periods of time are not clear.

The primary aim of this study was to investigate the baseline fasting plasma concentrations of intact proinsulin, 32-33 split proinsulin, specific insulin and IRI, for the development of Type 2 diabetes and to establish the role of AIR at an IVGTT in these associations. Further, possible significant predictors by outcome associations were investigated in terms of different length of follow-up, i.e. development of Type 2 diabetes 10, 20 and 27 years later.

\section{Subjects and methods}

Study design. The Uppsala Longitudinal Study of Adult Men is a population-based cohort study of diabetes and cardiovascular disease in men [6, 19]. In 1970, all men born between 1920 and 24 and residing in Uppsala were invited to a health survey at age 50 years (U-50), between 1970 and 1973 (baseline). In all, 2322 of 2841 invited men participated (82\%). Plasma samples have been stored since that survey, allowing us to study proinsulin, using highly specific assays, as a possible predictor for the development of Type 2 diabetes in non-diabetic subjects at baseline, using the follow-up data over 27 years.

Due to a freezer failure causing the random loss of some 1000 samples, concentrations of intact and 32-33 split proinsulin and true insulin were analysed in baseline plasma samples from 1306 subjects. There were no differences in baseline characteristics other than propeptides, between those with and those without their propeptide concentrations analysed, indicating that no selection bias had occurred due to the loss of samples [20].

Subjects. This study was based on those subjects who were non-diabetic at baseline and had complete data from the baseline investigation and proinsulin and specific insulin assessments and follow-up data from the 10-year follow-up $(n=777)$, from the 20-year follow-up $(n=436)$, and from the 27-year follow-up $(n=348)$. In order to select non-diabetic men at baseline, subjects with a fasting blood glucose greater than or equal to $6.1 \mathrm{mmol} / \mathrm{l}$ or treatment with oral hypoglycaemic agents (OHAs) were excluded. Subjects with insulin treatment only, indicative of Type 1 Diabetes mellitus, at baseline or at any follow-up were also excluded from the study.

Information concerning previous disease and current pharmacological treatment was collected using a medical questionnaire and through interviews conducted by one physician [19, 21].

Outcomes. The outcome variables, described below, were defined using the follow-up data from the second, third and fourth investigations (U-60, U-70 and U-77) carried out in the original Uppsala-cohort at age 60 years (U-60) between 1980 and 1984 (1860 subjects participated) [21], at age 70 years (U-70) between 1991 and 1995 (1221 out of 1681 eligible men participated) [22] and at age 77 years (U-77) between 1998 and 2001 (839 out of 1079 eligible men participated).

Type 2 diabetes was defined according to the World Health Organisation (WHO) criteria from 1999 [23] (using fasting concentrations) and/or use of OHAs. At the first two investigations (U-50 and U-60), fasting blood glucose concentrations were analysed and Type 2 diabetes was thus defined as a fasting blood glucose concentration greater than or equal to $6.1 \mathrm{mmol} / \mathrm{l}$ and/or treatment with OHAs. At the third and fourth investigations (U-70 and U-77), fasting plasma glucose concentrations were analysed and Type 2 diabetes was defined as a fasting plasma glucose concentration greater than or equal to $7.0 \mathrm{mmol} / \mathrm{l}$ and/or treatment with OHAs. The cumulative incidence of Type 2 diabetes was calculated, from age 50 years to age 60 years as Type 2 diabetes identified at U-60, from age 50 to age 70 as Type 2 diabetes identified at U-60 or U-70 and from age 50 years to age 77 years as Type 2 diabetes identified at U-60, U-70 or U-77.

To avoid potential bias by using only fasting glucose concentrations for the diagnosis of Type 2 diabetes, an additional analysis was carried out using data from a 75-gram OGTT, which was carried out in all participants in U-70. Type 2 diabetes was defined as a fasting plasma glucose greater than or equal to $7.0 \mathrm{mmol} / \mathrm{l}$ or a 2 -h glucose glucose greater than or equal to $11.1 \mathrm{mmol} / \mathrm{l}$, according to WHO criteria from 1999 [23] and/or treatment with OHAs.

Baseline characteristics and laboratory methods. The concentrations of intact and 32-33 split proinsulin were analysed using the two-site immunometric assay technique [24], in plasma samples that had been stored frozen $\left(-70^{\circ} \mathrm{C}\right)$ since baseline. Specific insulin concentrations were also analysed in these samples using the Access Immunoassay System (Sanofi Pasteur Diagnostics, Marmes-la-Coquette, France), using a onestep chemiluminescent immunoenzymatic assay. These procedures have been described in detail previously and the longterm storage did not interfere with the assessments in plasma that had been stored for 25 years [20].

At baseline, the serum IRI concentrations were analysed with the Phadebas Insulin Test (Pharmacia AB, Uppsala, Sweden) [19, 21].

An IVGTT, using a glucose dose of $0.5 \mathrm{~g} / \mathrm{kg}$ body weight, was carried out in 1792 men at baseline [19]. The serum IRI concentrations during the IVGTT were measured in duplicate blood samples drawn before and at 4 and 6 minutes after the start of the glucose injection. The AIR was expressed as the mean value of the serum insulin concentrations at 4 and 6 minutes.

Blood glucose was analysed, at U-50 and U-60 using spectrophotometry and the glucose oxidase method [19, 21]. At U-70 and U-77 plasma glucose was analysed by the glucose dehydrogenase method (Gluc-DH, Merck, Darmstadt, Germany).

Body mass index (BMI) was calculated as weight (in underwear) / height squared $\left(\mathrm{kg} / \mathrm{m}^{2}\right)$.

The study was approved by the Ethics Committee of the Faculty of Medicine at Uppsala University. Informed consent was obtained from all participants.

Statistical analyses. Skewed variables (insulin and propeptide measurements and glucose) were log transformed to achieve normal distribution. Normally distributed variables were used in all statistical analyses, done using the statistical software package Stata 6.0 for PC (Stata Corporation, College Station, Tex., USA). Logistic regression analyses were used on standardised variables (standardised to one SD) to assess the mag- 
nitude of the relationship to, and the statistical significance of the predictors of each of the defined outcomes. Results are shown as odds ratio with their $95 \%$ confidence intervals, i.e. all tests were two-tailed and a $p$ value of less than 0.05 was considered statistically significant. All analyses were adjusted for age at baseline and length of follow up between investigations.

In the multivariate models, analysing fasting intact and 32-33 split proinsulin, specific insulin and IRI one by one, adjustments were made for the possible confounding effects of AIR, BMI and fasting glucose as predictors for Type 2 diabetes by multiple logistic regression. Further adjustment for insulin was made for the associations between propeptides and Type 2 diabetes. To study possible multiplicative effects of significant predictors, interaction terms between significant predictors of Type 2 diabetes were tested within the multivariate models. The tertile limits of several factors were calculated in the whole baseline population to get reliable estimates and used in trend tests, using logistic regression, for the development of Type 2 diabetes. The limits were for: Intact proinsulin concentrations, tertile 1 (T1) less than $1.7 \mathrm{pmol} / 1$, tertile 2 (T2) greater than or equal to $1.7 \mathrm{pmol} / 1$ or less than $3.1 \mathrm{pmol} / 1$ and tertile 3 (T3) greater than or equal to $3.1 \mathrm{pmol} / \mathrm{l}$. For $32-33$ split proinsulin concentrations, T1 less than $4.3 \mathrm{pmol} / \mathrm{l}, \mathrm{T} 2$ greater than or equal to $4.3 \mathrm{pmol} / 1$ or less than $7.2 \mathrm{pmol} / \mathrm{l}, \mathrm{T} 3$ greater than or equal to $7.2 \mathrm{pmol} / \mathrm{l}$. For specific insulin concentrations, T1 less than $32 \mathrm{pmol} / \mathrm{l}, \mathrm{T} 2$ greater than or equal to $32 \mathrm{pmol} / \mathrm{l}$ or less than $53 \mathrm{pmol} / \mathrm{l}, \mathrm{T} 3$ greater than or equal to $53 \mathrm{pmol} / \mathrm{l}$. For AIR, T1 less than $282 \mathrm{pmol} / \mathrm{l}$, T2 greater than or equal to $282 \mathrm{pmol} / \mathrm{l}$ or less than $456 \mathrm{pmol} / \mathrm{l}$, T3 greater than or equal to $456 \mathrm{pmol} / \mathrm{l}$. For blood glucose concentration, T1 less than $4.6 \mathrm{mmol} / \mathrm{l}, \mathrm{T} 2$ greater than or equal to $4.6 \mathrm{mmol} / \mathrm{l}$ or less than $5.0 \mathrm{mmol} / \mathrm{l}$ and $\mathrm{T} 3$ greater than or equal to $5.0 \mathrm{mmol} / 1$. For BMI, T1 less than $23.5 \mathrm{~kg} / \mathrm{m}^{2} \mathrm{~T} 2$ greater than or equal to $23.5 \mathrm{~kg} / \mathrm{m}^{2}$ or less than $26.0 \mathrm{~kg} / \mathrm{m} 2$ and T3 greater than or equal to $26.0 \mathrm{~kg} / \mathrm{m}^{2}$.

\section{Results}

The cumulative incidence of Type 2 diabetes, during the course of the study was $5.8 \%(106 / 1834)$ from age 50 to 60 years; $19.9 \%$ (252/1263) from age 50 to 70 years and $35.2 \%$ (340/967) from age 50 to 77 years. Corresponding numbers for the subset with proinsulin assessments were $6.1 \%$ (48/777); $18.9 \%$ (88/466) and 33.0\% (115/348).

Clinical characteristics and crude odds ratios for the development of Type 2 diabetes, are shown in Table 1. Fasting intact and 32-33 split proinsulin, specific and immunoreactive insulin, as well as AIR, BMI and fasting blood glucose concentrations were all associated with development of Type 2 diabetes at the 10-year, the 20-year and the 27-year follow-up periods. Intact proinsulin and 32-33 split proinsulin showed stronger associations with the development of Type 2 diabetes than did specific insulin and IRI, as judged by the magnitude of the standardised odds ratios. After adjustment for specific insulin, the association between proinsulin and Type 2 diabetes remained significant. A high AIR was associated with a smaller risk for development of Type 2 diabetes over all three follow-up periods.

When adjusted for confounding effects of BMI and fasting glucose concentrations, only intact proinsulin and 32-33 split proinsulin showed a congruent pattern, as they were associated with the development of Type 2 diabetes over all three follow-up periods, independent of confounding factors. After adjustment, specific insulin predicted Type 2 diabetes only between ages 50 and 60 years and IRI did not predict Type 2 diabetes over any of the follow-up periods.

The results from separate multiple logistic regression models, are shown in Table 2 for intact proinsulin (model 1) and for 32-33 split proinsulin where, in both settings, AIR, BMI and fasting glucose were included within each model. Both intact and 32-33 split proinsulin were associated with the development of Type 2 diabetes over all three periods of follow-up. An attenuating effect by the length of follow-up time was observed.

Decreased AIR showed an association with Type 2 diabetes which was unchanged with time of followup, whereas increased BMI tended to be more strongly associated with development of Type 2 diabetes for

Table 1. Clinical characteristics at baseline and odds ratios for the development of Type 2 diabetes

\begin{tabular}{lclll}
\hline & Means \pm SD & $\begin{array}{l}\text { Type 2 DM } \\
\text { Age 50-60 }\end{array}$ & $\begin{array}{l}\text { Type 2 DM } \\
\text { Age 50-70 } \\
\text { Odds Ratio (95\% CI) }\end{array}$ & $\begin{array}{l}\text { Type 2 DM } \\
\text { Age 50-77 }\end{array}$ \\
& & & Odds Ratio (95\% CI) & Odds Ratio (95\% CI) \\
\hline Intact proinsulin (pmol/l) & $2.9 \pm 2.6$ & $2.63(1.89-3.67)$ & $2.52(1.86-3.40)$ & $1.87(1.46-2.39)$ \\
Intact proinsulin adjusted for specific insulin & - & $2.22(1.52-3.25)$ & $2.07(1.48-2.90)$ & $1.52(1.15-2.01)$ \\
32-33 split proinsulin (pmol/l) & $6.9 \pm 6.6$ & $2.42(1.79-3.29)$ & $2.46(1.82-3.34)$ & $1.97(1.52-2.50)$ \\
32-33 split proinsulin adjusted for specific & - & $2.14(1.40-3.27)$ & $2.11(1.42-3.14)$ & $1.59(1.15-2.21)$ \\
$\quad$ insulin & $49.0 \pm 35.6$ & $2.03(1.50-2.74)$ & $2.08(1.57-2.76)$ & $1.79(1.37-2.34)$ \\
Specific insulin (pmol/l) & $75.6 \pm 43.5$ & $1.92(1.43-2.57)$ & $2.23(1.67-2.98)$ & $1.72(1.36-2.17)$ \\
Fasting IRI (pmol/l) & $416 \pm 265$ & $0.47(0.33-0.66)$ & $0.59(0.46-0.78)$ & $0.66(0.52-0.84)$ \\
AIR (pmol/l) & $4.9 \pm 0.5$ & $2.44(1.79-3.32)$ & $2.30(1.72-3.10)$ & $2.00(1.54-2.61)$ \\
Fasting blood glucose (mmol/l) & $24.9 \pm 3.1$ & $1.99(1.54-2.59)$ & $2.50(1.87-3.35)$ & $2.05(1.59-2.66)$ \\
BMI (kg/m $\left.{ }^{2}\right)$ & & &
\end{tabular}

Arithmetic means \pm SD. Odds Ratios from logistic regression were applied to variables standardised to $1 \mathrm{SD}$ and adjusted for age at entry 
Table 2. Multivariate logistic regression models Type 2 diabetes as the outcome

\begin{tabular}{llll}
\hline & $\begin{array}{c}\text { Type 2 DM Age 50-60 } \\
\text { Odds Ratio (95\%-CI) }\end{array}$ & $\begin{array}{l}\text { Type 2 DM Age 50-70 } \\
\text { Odds Ratio (95\%-CI) }\end{array}$ & $\begin{array}{c}\text { Type 2 DM Age 50-77 } \\
\text { Odds Ratio (95\%-CI) }\end{array}$ \\
\hline Model 1 & & & $1.53(1.20-1.94)$ \\
Proinsulin & $2.25(1.40-3.59)$ & $0.48(0.33-0.69)$ & $1.57(1.16-2.14)$ \\
AIR & $0.45(0.30-0.66)$ & $1.67(1.29-2.16)$ & $2.18(1.54-3.09)$ \\
BMI & $1.53(1.06-2.21)$ & $1.29(1.03-1.62)$ & $1.56(1.15-2.12)$ \\
Fasting blood glucose & $1.63(1.13-2.36)$ & $1.43(1.11-1.84)$ & $1.35(1.01-1.88)$ \\
Model 2 & & & $1.40(1.08-1.82)$ \\
Proinsulin also adjusted for specific insulin & $1.85(1.13-3.01)$ & $0.47(0.33-0.67)$ & $1.70(1.20-2.39)$ \\
Model 3 & & $1.67(1.28-2.17)$ & $0.50(0.37-0.69)$ \\
32-33 split proinsulin & $2.18(1.35-3.54)$ & $1.27(1.01-1.59)$ & $1.04(1.43-2.93)$ \\
AIR & $0.45(0.31-0.66)$ & & $1.48(1.09-2.00)$ \\
BMI & $1.45(0.98-2.13)$ & $1.25(0.93-1.66)$ & $1.29(0.87-1.91)$ \\
Fasting blood glucose & $1.61(1.11-2.32)$ & & \\
Model 4 & & &
\end{tabular}

Odds Ratios from logistic regression were applied to variables standardised to $1 \mathrm{SD}$ and adjusted for age at entry

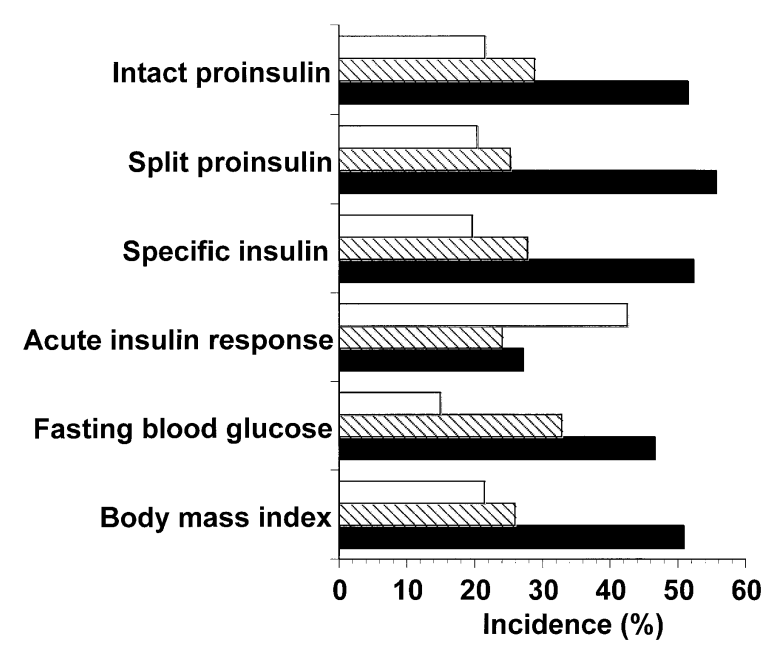

Fig. 1. Cumulative incidence of Type 2 diabetes over 27 years of follow-up from age 50, by tertiles of intact and 32-33 split proinsulin and specific insulin, acute insulin response (AIR) at an intravenous glucose tolerance test, BMI and fasting glucose concentrations $(n=348)$. T denotes tertile. Test for trend was significant in all cases, $p<0.001$

longer times of follow-up. Both intact and 32-33 split proinsulin were predictors, independent of AIR, over all three follow-up periods. The association between intact proinsulin and conversion to Type 2 diabetes was not altered by further adjustment for specific insulin (model 2). However, partially processed proinsulin (32-33 split proinsulin) was no longer a statistically significant predictor of Type 2 diabetes when further adjusted for specific insulin (model 4).

There was a pronounced increase in the incidence of Type 2 diabetes over the 27 years in the third tertile of intact and 32-33 split proinsulin, specific insulin,

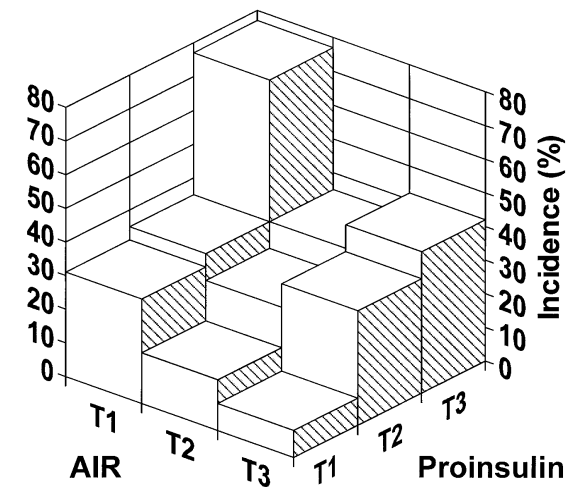

Fig. 2. Cumulative incidence of Type 2 diabetes over 27 years of follow-up from age 50 by tertiles of proinsulin concentrations and tertiles of Acute insulin response (AIR). The interaction between proinsulin and AIR was significant when tested in multiple logistic regression, $p=0.004$

fasting blood glucose and BMI and the first tertile of AIR (Fig. 1).

The interaction term between intact proinsulin and AIR was related to the development of Type 2 diabetes when tested for $(\mathrm{p}<0.05$; Fig. 2). A high incidence of $77 \%$ for Type 2 diabetes was shown in the group of subjects in the high tertile of proinsulin and the low tertile of AIR, illustrating a multiplicative effect of a concurrent high proinsulin concentration and a low AIR. The relative risk for Type 2 diabetes in the group defined by the high tertile of proinsulin and the low tertile of AIR in comparison with the contrast group defined by the low tertile of proinsulin and the high tertile of AIR was $9.23(95 \%$-CI, 6.49-11.97). 
In the additional analysis, when Type 2 diabetes was diagnosed based on the 2-h glucose concentrations from the OGTT at age 70 years also, the results were found to be essentially identical to those shown above.

\section{Discussion}

Both fasting intact and fasting 32-33 split proinsulin predicted development of Type 2 diabetes, over a 10 -year, 20 -year and a 27 -year follow-up in this long term population-based study of men confirming results from some earlier studies with follow-up times of less than 7 years $[11,12,13,14,15,16]$. The associations were independent of the effect of a low AIR after an intravenous glucose load. The risk for Type 2 diabetes increased when the fasting intact proinsulin exceeded a concentration of $3.1 \mathrm{pmol} / \mathrm{l}$ (the third tertile) in middle age, particularly when concurrent with a low AIR. Specific insulin was not a long-term predictor in the multivariate analyses whereas intact proinsulin was a predictor.

High proinsulin and low AIR were found to interact as predictors, for the development of Type 2 diabetes. When both abnormalities occur, a multiplicative effect of them generates a very high risk of $77 \%$ risk to develop Type 2 diabetes (Fig. 2). The results suggest that high proinsulin and low AIR mirror different aspects of beta-cell dysfunction, given the reservation that the association between proinsulin and insulin resistance was not taken into account.

Insulin resistance and insulin secretory dysfunction are independent predictors of glucose tolerance at any stage of the development of Type 2 diabetes [3]. In terms of being a predictor for the development of Type 2 diabetes, fasting proinsulin could be considered as a measurement of insulin secretory dysfunction influenced by both insulin sensitivity and the degree of a pancreatic beta-cell defect.

Hyperproinsulinaemia could be a sign of a pancreatic beta-cell defect that is augmented by an increased demand placed on the beta cell by hyperglycaemia [25]. However, in this study, proinsulin was still a predictor of Type 2 diabetes independent of fasting blood glucose concentrations. It has also been suggested that hyperproinsulinaemia is the result of secretion of immature proinsulin-rich granules from beta cells, as a response to an increased demand for insulin [26], i.e. an insulin resistant state.

Furthermore, low insulin secretion in itself, assessed as the response to intravenous arginine under maximum glycaemic potentiation, is associated with disproportionately increased proinsulin concentrations [27], an observation, which could explain the interaction, discussed above, between high proinsulin and beta-cell dysfunction, assessed as a low AIR, in predicting Type 2 diabetes.

The associations between proinsulin and Type 2 diabetes were adjusted for specific insulin concentra- tions in this study. Some previous studies have analysed the proinsulin to insulin ratio, which was a predictor of Type 2 diabetes in two $[13,16]$ but not in our or in four other studies $[11,12,14,15]$. The inconsistencies might be due to reduced precision when using a ratio between two biological variables [28].

Our results show that increased plasma proinsulin precedes the onset of Type 2 diabetes by up to 27 years, independent of BMI. In concert with this observation, hyperproinsulinaemia was not explained by obesity-associated insulin resistance in subjects with Type 2 diabetes [29]. The waist-to-hip ratio could be used as an index of central obesity. However these data were not collected at baseline.

Intact proinsulin was a stronger predictor for Type 2 diabetes than specific insulin. Insulin has a considerably shorter half-life than proinsulin [24] and insulin concentrations might be more affected by the oscillations of insulin secretion [30], both contributing to the lower stability (i.e. the precision of a point estimate of continuously varying plasma concentrations) of insulin measurements compared to proinsulin measurements. Proinsulin could thus have a better predictive capacity than insulin, assuming that both have the same effect on the outcome.

The relationship between specific insulin and development of Type 2 diabetes was weaker, but still important over 10 but not over 20 or 27 years of followup, when adjusted for BMI and fasting glucose concentrations, consistent with previous studies [14, 15].

Our findings suggest that immunoreactive insulin assays used in many earlier studies could have overestimated the strength of the association between insulin, i.e. IRI, and development of Type 2 diabetes. Our observations support the idea that an increase in concentrations of insulin precursors constitutes the association with Type 2 diabetes rather than the plasma insulin concentrations per se.

The IVGTT, not widely used today, was at the time for the baseline investigation (1970) considered to be a useful method for diagnosing diabetes, before no broadly accepted diagnostic criteria for diabetes by OGTT were available [31]. We used data from the IVGTT only for the assessment of AIR and fasting concentrations of insulin propeptides and glucose. Subjects were identified having Type 2 diabetes by the fasting glucose concentration and use of OHAs. Men with diabetes at baseline were excluded. The current diagnostic criteria for diabetes, based on the 2-h glucose concentration after an OGTT or the fasting glucose concentration do not fully match [32] such that subjects fulfilling the $2-\mathrm{h}$ criterion could be classified as non-diabetic based on the fasting glucose concentrations. This might have attenuated the observed associations between insulin propeptides and Type 2 diabetes and not overestimated them. The results for prediction of Type 2 diabetes were essentially identical when diabetes was diagnosed at age 70 based on 
the 2-h glucose concentration at the OGTT as well as of the fasting glucose concentration.

The loss of baseline samples reduced the size of the study population and might diminish the power of the study. However, no selection bias was found when it was tested for. In addition, the size of the study sample was large enough to give us the power of showing an $(p=0.004)$ interaction between proinsulin and AIR. Some selection by death is likely to have occurred over this long follow-up and may have weakened the relationship between proinsulin and development of Type 2 diabetes. Proinsulin [20], like Type 2 diabetes [33], is a predictor of cardiovascular death whereby a number of subjects in the higher range of plasma proinsulin will probably have been lost to follow up.

We conclude that proinsulin is a strong and a statistically significant predictor of the development of Type 2 diabetes, mirroring both beta-cell dysfunction and insulin resistance. Further, proinsulin was predicting Type 2 diabetes particularly strongly in subjects with a low acute insulin response at baseline. Furthermore, proinsulin is a better predictor of Type 2 diabetes than specific or immunoreactive insulin.

Acknowledgements. Supported by research grants from The Medical Faculty of Uppsala University, the Swedish Medical Research Council (MFR5446), the Foundation for Geriatric Research, The Swedish National Association against Heart and Lung Disease, the Swedish Diabetes Association Research Fund, the Uppsala Geriatric Fund, the Swedish Council for Planning and Co-ordination of Research, Ernfors Fund for Diabetes Research, the Thureus Foundation for Geriatric Research and the Medical Research Council, UK.

We thank the technical staff of the Department of Clinical Biochemistry, Addenbrooke's Hospital for the measurements of specific insulin, intact and 32-33 split proinsulin.

\section{References}

1. Gerich JE (1998) The genetic basis of type 2 diabetes mellitus: impaired insulin secretion versus impaired insulin sensitivity. Endocr Rev 19:491-503

2. Lillioja S, Mott DM, Spraul M et al. (1993) Insulin resistance and insulin secretory dysfunction as precursors of non-insulin-dependent diabetes mellitus. Prospective studies of Pima Indians. N Engl J Med 329:1988-1992

3. Weyer C, Tataranni PA, Bogardus C, Pratley RE (2001) Insulin resistance and insulin secretory dysfunction are independent predictors of worsening of glucose tolerance during each stage of type 2 diabetes development. Diabetes Care 24:89-94

4. Saad MF, Knowler WC, Pettitt DJ, Nelson RG, Mott DM, Bennett PH (1988) The natural history of impaired glucose tolerance in the Pima Indians. N Engl J Med 319:15001506

5. Haffner SM, Miettinen H, Gaskill SP, Stern MP (1995) Decreased insulin secretion and increased insulin resistance are independently related to the 7-year risk of NIDDM in Mexican-Americans. Diabetes 44:1386-1391

6. Skarfors ET, Selinus KI, Lithell HO (1991) Risk factors for developing non-insulin dependent diabetes: a 10 year follow up of men in Uppsala. BMJ 303:755-760
7. Charles MA, Fontbonne A, Thibult N, Warnet JM, Rosselin GE, Eschwege E (1991) Risk factors for NIDDM in white population. Paris prospective study. Diabetes 40:796-799

8. Mykkanen L, Kuusisto J, Pyorala K, Laakso M (1993) Cardiovascular disease risk factors as predictors of type II (non- insulin-dependent) diabetes mellitus in elderly subjects. Diabetologia 36:553-559

9. Saad MF, Anderson RL, Laws A et al. (1994) A comparison between the minimal model and the glucose clamp in the assessment of insulin sensitivity across the spectrum of glucose tolerance. Insulin Resistance Atherosclerosis Study. Diabetes 43:1114-1121

10. Laakso M (1993) How good a marker is insulin level for insulin resistance? Am J Epidemiol 137:959-965

11. Hanley AJ, D’Agostino R Jr, Wagenknecht et al. (2002) Increased proinsulin levels and decreased acute insulin response independently predict the incidence of type 2 diabetes in the insulin resistance atherosclerosis study. Diabetes $51: 1263-1270$

12. Wareham NJ, Byrne CD, Williams R, Day NE, Hales CN (1999) Fasting proinsulin concentrations predict the development of type 2 diabetes. Diabetes Care 22:262-270

13. Haffner SM, Gonzalez C, Mykkanen L, Stern M (1997) Total immunoreactive proinsulin, immunoreactive insulin and specific insulin in relation to conversion to NIDDM: the Mexico City Diabetes Study. Diabetologia 40:830837

14. Nijpels G, Popp-Snijders C, Kostense PJ, Bouter LM, Heine RJ (1996) Fasting proinsulin and 2-h post-load glucose levels predict the conversion to NIDDM in subjects with impaired glucose tolerance: the Hoorn Study. Diabetologia 39:113-118

15. Kahn SE, Leonetti DL, Prigeon RL, Boyko EJ, Bergstrom RW, Fujimoto WY (1995) Proinsulin as a marker for the development of NIDDM in Japanese-American men. Diabetes 44:173-179

16. Mykkanen L, Haffner SM, Kuusisto J, Pyorala K, Hales CN, Laakso M (1995) Serum proinsulin levels are disproportionately increased in elderly prediabetic subjects. Diabetologia 38:1176-82

17. Mykkanen L, Haffner SM, Hales CN, Ronnemaa T, Laakso M (1997) The relation of proinsulin, insulin, and proinsulin-to-insulin ratio to insulin sensitivity and acute insulin response in normoglycemic subjects. Diabetes 46:19901995

18. Mykkanen L, Zaccaro DJ, Hales CN, Festa A, Haffner SM (1999) The relation of proinsulin and insulin to insulin sensitivity and acute insulin response in subjects with newly diagnosed Type II diabetes: the Insulin Resistance Atherosclerosis Study. Diabetologia 42:1060-1066

19. Hedstrand H (1975) A study of middle-aged men with particular reference to risk factors for cardiovascular disease. Ups J Med Sci 80 [Suppl 19]:1-61

20. Zethelius B, Byberg L, Hales CN, Lithell H, Berne C (2002) Proinsulin is an independent predictor of coronary heart disease: report from a 27-year follow-up study. Circulation 105:2153-2158

21. Skarfors ET, Lithell HO, Selinus I (1991) Risk factors for the development of hypertension: a 10-year longitudinal study in middle-aged men. J Hypertens 9:217-223

22. Lithell H, Sundstrom J, Arnlov J et al. (2000) Epidemiological and clinical studies on insulin resistance and diabetes. Ups J Med Sci 105:135-150

23. World Health Organisation (1999) Definition, diagnosis and classification of diabetes mellitus and its complications: report of a WHO consultation. WHO/NCD/NCS/99.2 World Health Organization, Geneva 
24. Sobey WJ, Beer SF, Carrington CA, Clark PMS, Hales CN (1989) Sensitive and specific two-site immunoradiometric assays for human insulin, proinsulin, 65-66 split and 32-33 split proinsulins. Biochem J 260:535-541

25. Rhodes CJ, Alarcon C (1994) What beta-cell defect could lead to hyperproinsulinemia in NIDDM? Some clues from recent advances made in understanding the proinsulin-processing mechanism. Diabetes 43:511-517

26. Yoshioka N, Kuzuya T, Matsuda A, Taniguchi M, Iwamoto Y (1988) Serum proinsulin levels at fasting and after oral glucose load in patients with Type 2 (non-insulin-dependent) diabetes mellitus. Diabetologia 31:355-360

27. Roder ME, Porte D Jr, Schwartz RS, Kahn SE (1998) Disproportionately elevated proinsulin levels reflect the degree of impaired B cell secretory capacity in patients with noninsulin-dependent diabetes mellitus. J Clin Endocrinol Metab 83:604-608

28. Allison DB, Paultre F, Goran MI, Poehlman ET, Heymsfield SB (1995) Statistical considerations regarding the use of ratios to adjust data. Int $\mathrm{J}$ Obes Relat Metab Disord 19:644-652
29. Roder ME, Dinesen B, Hartling et al. (1999) Intact proinsulin and beta-cell function in lean and obese subjects with and without type 2 diabetes. Diabetes Care 22:609-614

30. Lang DA, Matthews DR, Peto J, Turner RC (1979) Cyclic oscillations of basal plasma glucose and insulin concentrations in human beings. N Engl J Med 301:1023-1027

31. World Health Organisation (1980) WHO Expert Committee on Diabetes Mellitus: second report. Technical Report Series No 646 World Health Organisation, Geneva

32. (No authors listed) (1998) Will new diagnostic criteria for diabetes mellitus change phenotype of patients with diabetes? Reanalysis of European epidemiological data. DECODE Study Group on behalf of the European Diabetes Epidemiology Study Group. BMJ 317:371-375

33. Khaw KT, Wareham N, Luben R et al. (2001) Glycated haemoglobin, diabetes, and mortality in men in Norfolk cohort of european prospective investigation of cancer and nutrition (EPIC-Norfolk). BMJ 322:15-18 